\title{
He 2-147: A case in which the expansion parallax method fails
}

\section{Miguel Santander-García ${ }^{1}$, Romano L. M. Corradi ${ }^{2,1}$, Patricia A. Whitelock $^{3,4}$, Ulisse Munari ${ }^{5}$, Antonio Mampaso ${ }^{1}$, Freddy Marang ${ }^{3}$, Francesca Boffi ${ }^{6}$, and Mario Livio ${ }^{6}$}

${ }^{1}$ Instituto de Astrofísica de Canarias, 38200 La Laguna, Tenerife, Spain, email: miguelsg@iac.es

${ }^{2}$ Isaac Newton Group of Telescopes, Ap. de Correos 321, 38700 Sta. Cruz de la Palma, Spain

${ }^{3}$ South African Astronomical Observatory, PO Box 9, 7935 Observatory, South Africa

${ }^{4}$ Department of Mathematics and Applied Mathematics and Department of Astronomy, University of Cape Town, South Africa

${ }^{5}$ INAF Osservatorio Astronomico di Padova, via dell'Osservatorio 8, 36012 Asiago (VI), Italy

${ }^{6}$ Space Telescope Science Institute, 3700 San Martin Drive, Baltimore, MD 21218, USA

\begin{abstract}
HST multi-epoch images and VLT integral-field, high resolution spectroscopy allowed us a robust determination of the 3 -D geometry and orientation of the nebula surrounding He 2147, and to measure its apparent expansion in the plane of the sky. Applying the expansion parallax method results in a distance which is significantly shorter that the one obtained via the period-luminosity (P-L) relationship for the Mira component. We show how allowing for shock excitation of the nebula, as suggested by the extremely broad nebular line profiles, enables both distance derivations to be reconciled.
\end{abstract}

Keywords. stars: symbiotic binaries, He 2-147, interstellar medium: kinematics and dynamics

HST WFPC2 F658N images, obtained in 2001 (Fig. 1 top left) and 2004, allow us to compute the apparent expansion of the nebula in the plane of the sky. Residuals of the subtraction of the two images reveal that the nebula has grown $0.15 \%$ in 3 years. At the same time, Doppler shift analysis of the $\mathrm{H} \alpha$ and [NII] lines of the VLT high resolution $(\mathrm{R}=27000)$ Integral Field Spectra (Fig. 1 bottom left) reveals an expansion pattern which is well fitted by a ring of gas inclined $\sim 68^{\circ}$ and expanding at $\sim 90 \mathrm{~km} \mathrm{~s}^{-1}$ (Fig. 1 top right). However, local departures from such an homologous expansion are present in the nebular region at P.A. $\sim 280^{\circ}$. In addition, line profiles have a remarkably large $\left(\sim 160 \mathrm{~km} \mathrm{~s}^{-1}\right)$ full width at zero intensity (see Fig. 1 bottom right) all over the nebula.

The expansion parallax method determines the distance by comparing the apparent expansion in the plane of the sky with the velocity of the gas along the line of sight, once the intrinsic geometry, orientation and kinematics of the nebula have been recovered by spatio-kinematical modeling. In the case of He 2-147, a straightforward calculation of the expansion parallax results in a distance of $1.71 \pm 0.31 \mathrm{kpc}$.

Alternatively, by assuming that the Mira in He 2-147 has similar characteristics to its solitary counterparts, it is possible to derive a distance from the P-L relation (Feast et al. 1989). NIR photometric data taken at the SAAO over 25 years reveal a pulsational period of 373 days for the Mira, and a $\mathrm{K}$ magnitude $K_{0}=4.45$ (corrected for a reddening $\mathrm{E}(\mathrm{B}-\mathrm{V})$ of 0.92$)$. The P-L relation gives $\mathrm{M}_{K}=-7.94$, implying a distance of $3.0 \pm 0.4 \mathrm{kpc}$, significantly larger than obtained from the expansion parallax.

However, the two measurements can be reconciled if one considers the very broad [NII] profiles (Fig. 1 Bottom Right) to be produced in a $160 \mathrm{~km} \mathrm{~s}^{-1}$ shock passing through a 

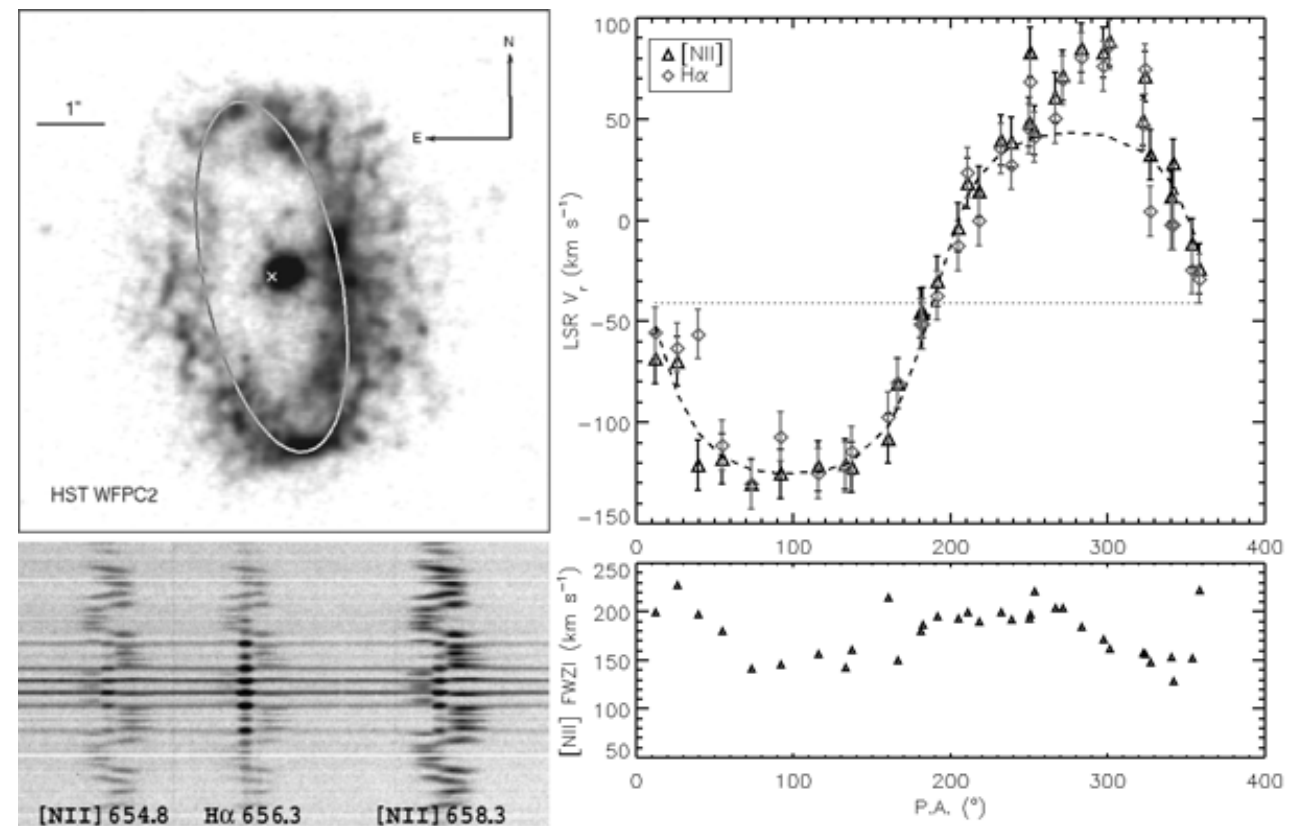

Figure 1. Top Left: HST [NII] WFPC2 2001 image of He 2-147. A fit to the nebular geometry is indicated by the ellipse. Bottom Left: The VLT + ARGUS spectra. Each row is the spectrum of one of the 314 fibres of the ARGUS Integral Field Unit. Top Right: LSR radial velocity of the barycentre of both $\mathrm{H} \alpha$ and [NII] emission lines of the nebula. The dashed line represents the expanding ring model, while the dotted line indicates LSR radial velocity of the core. Bottom Right: Full width at zero intensity (FWZI) of the [NII] data.

partially ionised medium. Such a shock is consistent with the high [NII] to $\mathrm{H} \alpha$ flux ratio found throughout the nebula (up to 7.7), and with the FWZI of the profiles according to the bow-shock models of Hartigan et al. (1987). Thus, the apparent expansion in the plane of the sky would not reflect gas motions, but rather the expansion of the shock front. This changes the derivation of the distance through the expansion-parallax method (see e.g. Mellema 2004), which must be scaled accordingly (by a factor of $\mathrm{V}_{\text {shock }} / \mathrm{V}_{r}=1.78$ ), to a value of $3.0 \pm 0.5 \mathrm{kpc}$, which is in good agreement with the figure found through the Mira P-L relation. A full discussion is given in Santander-García et al. (in preparation).

He 2-147 illustrates both the potential virtues and problems of the expansion parallax method for determining distances in PNe. In the presence of shocks, a straightforward application of the method can severely underestimate distances. This is the case for He 2-147, where we have found evidence for a strong shock front passing through the nebula. By applying two independent methods (shock-corrected expansion parallax and Mira P-L relation), we have derived a distance of $3.0 \pm 0.5 \mathrm{kpc}$.

\section{References}

Feast, M. W., Glass, I. S., Whitelock, P. A. \& Catchpole, R. M. 1989, MNRAS 241, 375

Hartigan, P., Raymond, J. \& Hartmann, L. 1987, ApJ 316, 323

Mellema, G. 2004, A\&ऽA 416, 623 